\title{
Selected Abstracts for 6th World Congress
}

\author{
WAEDM Task Force 01 \\ International Medical Aid in Disaster \\ Chairman: Steve Rottman \\ Task Force 1.1 \\ Monday, 11 September, 10:30-12:30: \\ Theatre 2
}

\section{TF 1.1.2}

An Advanced Training and Clinic Program for Medics in War-Torn Afghanistan

Robert R Simon

Professor, Department of Emergency Medicine, Cook County

Hospital, Chicago, Ill., USA

This paper will provide a synoptic history of the International Medical Corps (IMC), of which the author is the Medical Director. It will outline the IMC's mission and goals with emphasis on its role in situations where neither government nor the traditional relief agencies are able to provide medical care within an active war zone. The presentation sets out the establishment of relief projects and clinics, and, in particular, the establishment of medic training programs for indigenous personnel.

The goal of such a program is to provide immediate care for some $80 \%$ of diseases and injuries on a self-reliance basis utilizing indigenous recruits. The backgrounds of applicants are described along with the screening, medical, surgical, laboratory, clinical, and public health training, and the unique clinical logistics and operational experience.

The establishment and operation of such a system within this austere setting may serve both as precedent and model for similar systems to be set up in adverse circumstances in the future, and perhaps as a model for augmentation of sparse, existing EMS systems in many parts of the developing world.

\author{
WAEDM, Task Force 2 \\ Hazardous Materials \\ Chairman: Craig Thrasher \\ Task Force 2.1 \\ Monday, 11 September, 14:00-15:30: Convention Hall
}

\section{TF 2.1.2}

Swedish National Board of Health and Welfare Guidelines on Toxic Chemical Incidents: A Basis for Worldwide Partnerships Per Kulling

Swedish Poison Information Centre, Stockholm, Sweden

The Emergency and Disaster Committee of the Swedish National Board of Health and Welfare has developed guidelines to enable health care systems to take preventive and interventive actions and reactions in response to hazardous toxic materials incidents. These guidelines were developed utilizing experience and databases from national and international studies and conferences, from which the characteristics of hazardous incidents were identified. From these characteristics, predictive scenarios were analyzed and used as a basis for recommendations on protocols for the identification of risk factors in both fixed and mobile sources of potential hazardous toxic materials. The guidelines set out methodology for both preemptive establishment of communication and command structures; the operational organization of administrative and intervening echelons is addressed, with emphasis placed on protocols for training and equipment of both administrative and rescue team personnel. Accent is placed on the establishment of fixed and responsive systems of data management, highlighting the imperatives for these systems to be capable of providing rapid, accurate, and precisely vectored incident information. Recommendations are made for the provision of fixed decontamination facilities at hospitals and for the provision of appropriate mobile decontamination with the capacity for deployment to the site of an incident, and augmented by the provision of guidelines for supplies of antidotes and medications. The need for prospective provision of the means to register and document incident and victim information is stressed with emphasis on the acquisition of data on demography, toxicology, and pathophysiology. In order to develop and implement action-oriented goals for dealing with the problem of major hazardous materials incidents for the WAEDM, a review of the interested worldwide organizations and their agenda will be presented. This summary will define the principle focus of each organization and compare it to all of the major elements of the solution. This comparison will suggest areas where the WAEDM should focus action and will serve as guidance for the derivation of recommendations to the General Assembly of WAEDM by the International Collaboration in Hazardous Materials Incidents. 
Symposium Session 01

Polytrauma 1, System Evaluation
Chairman: Howard Champion
Moderator: PC Leung
Monday, 11 September, 14:00-15:30:
Theatre 1

S 1.3

Partition: Evaluation of Prehospital Trauma

William J Sacco, WS Copes, LW Brain, DM Dove, and

Howard R Champion

Washington Hospital Center, Washington, DC, USA, and TriAnalytics, Inc., Bel Air, Maryland, USA

PARTITION, a method for evaluating prehospital services for trauma patients, is presented. PARTITION can be used to compare the effectiveness of a particular service to a norm based on the accumulated experience of many prehospital care providers. Here, the norm is a logistic function relating survival probability to a weighted combination of scene and admission Revised Trauma Score values, Injury Severity Score, and patient age from 18,841 direct admissions to the Major Trauma Outcome Study (MTOS) (9.6\% mortality rate) submitted through 1987 . The PARTITION norm discriminated well between survivors and deaths, as measured by a relative information gain $(R)$ value of 0.89 , a disparity $(D)$ value of 0.71 , and a mis-classification rate of $3.3 \%$. The average norm-predicted survival probabilities for survivors and deaths were 0.98 and 0.27 respectively. The PARTITION norm can be used to estimate the expected increase in the number of survivors, attributable to a prehospital service, over and above the number expected to survive a baseline (MTOS) hospitals providing baseline prehospital care.
Symposium Session $\mathrm{O} 2$

Cardiology 1, The Continuum of Care

Chairman: Richard Cummins

Moderator: CH Cheng

Monday, 11 September, 14:00-15:30:

Theatre 2

\section{S 2.5}

Gitizen CPR Teaching: The Belgian Experience

Leon Bossaert, $R$ Van Hoeyweghen, and the Steering Comittee. of the CPR Campaign " 3 minutes for a life"

University Antwerp-UIA, Belgium

In 1986-88, the Belgian Red Cross and the Belgian Heart Association organized a nationwide citizen-CPR campaign. One hundred thousand citizens were trained in a standardized, single, three-hour course.

\section{OBJECTIVES AND METHODS:}

1) To evaluate the promotional campaign and to determine the attitudes of the Belgian population towards CPR, utilizing two telephone inquiries with a one year interval; 2) To determine whether the CPR trainee could match the bystander performance at a cardiac arrest (CA), as described in the registry of the Belgian Cerebral Resuscitation study group (the CA registry); 3) To evaluate teaching results and attitudes of CPR trainees and how they were influenced by characteristics of trainee, instructor; and course; and 4) To determine teaching results of instructors with more or less CPR teaching experience.

\section{RESULTS:}

The telephone inquiry $(n=2400)$ indicated that citizens willing to attend a CPR course were young ( $<45 \mathrm{yrs:} 70 \%$ ), well educated (at least upper high school: $76 \%)$, sportive (53\%), employee or executive $(44 \%)$.

CPR trainees (study sample size $=17,491)$ were young $(71 \%$ aged $<40)$, mostly students $(33 \%)$ and employees $(31 \%)$, with a high likelihood of having had previous CPR training experience $(38 \%)$. The CA registry $(n=3083)$ has shown that $\mathrm{CA}$ usually happens in patients aged $>50 \mathrm{yrs}(76 \%)$, at home $(69 \%)$ and during office hours $(71 \%)$. Therefore, only $24 \%$ of CPR trainees belonged to the group most likely to be on the scene of CA.

Trainees, trained by experienced instructors (49\%), reached teaching objectives ( $>80 \%$ correct) in $79 \%$ for the cognitive test and in $95 \%$ for the practical test; those trained by less experienced instructors $(51 \%)$ in $82 \%$ and $96 \%$ respectively.

\section{CONGLUSIONS:}

These results indicate that in mass CPR programs, teaching objectives can be reached in a three-hour course, that promotion and instruction should be directed towards specific target groups, and that teaching results are equally good for instructors with more or less CPR teaching experience. 
Task Force 2.2

Monday, 11 September, 16:00-17:30: Convention Hall

\section{S 2.6}

Travel Light, Travel Fast: Motorcycle Paramedics in Hong Kong

\author{
Michael Moles \\ Anaesthetic Unit, Department of Maxillofacial Surgery and \\ Medicine, Prince Philip Dental Hospital, University of Hong \\ Kong
}

There is now unequivocal evidence of improved survival and outcome from acute cardiorespiratory emergencies related to the time interval from incident to first response, and whether Basic Life Support or Advanced Life Support have been provided. Preferably, this should be provided by bystander citizens trained in a public program; irrespective however, the response time should be less than nine minutes for second or first responder paraprofessionals, Ambulancemen, EMTs, or Paramedics. The less the incidence or the quality of citizen CPR, the more the need for rapid response.

The current strategy for achievement of these rapid responses is to establish a dense cellular system of responder stations; this is very costly, and invariably is penalized by circadian variations in traffic densities when attempting to provide rapid response times during periods of high density. In Hong Kong, an alternative strategy is under trial. Ambulancemen of the Fire/Ambulance Service have been equipped with police-type motorcycles specially fitted with panniers to carry Basic Life Support equipment. The motorcycles were provided by the Royal Hong Kong Police and the equipment substantially funded by local charitable organizations; no government funding direct or indirect has been allotted in support of the project. A pilot study of rapid response logistics was carried out using data culled from the encoder systems fitted to Ambulances in Hong Kong which enable time-activity-manning status to be transmitted by telemetry into the Ambulance Command computer. The study was used to identify catchment areas of high rapid-response load and the motorcycles located accordingly. Dispatch of the motorcycle units is at the discretion of the Ambulance Controllers and operates in two modes: either an Ambulance is dispatched and if held up in traffic, they then call for motorcycle back-up; or a motorcycle and Ambulance are dispatched synchronously. The dispatch, mobilization, transit, parking, and deployment to first response for the motorcycles, also fitted with encoders, invariably are more rapid than that for ambulances. If, as is usual in local the traffic conditions, the motorcycle "paramedic" arrives first, he can deliver and maintain Basic Life Support (or could with appropriate medical support and training, deliver Advanced Life Support) in the period until the ambulance arrives to remove and transport the victims in the conventional manner. This remarkably simple system, harnessed to proven outcome benefit and to costeffectiveness is applicable either in high density urban or in remote rural areas and merits consideration as an in tegral component of all emergency medical services systems in the pursuit of better response times. It may be speculated that the presence of such an echelon in the system easily could be used to increase public awareness of the complementary value of rapid citizen first response, thereby forging together most effectively, the first two links in the chain of care.

\section{TF 2.2.3}

\section{Hospital Planning and Training for Toxicological Mass Casualties}

Yehuda L Danon and Z Tuchner

Medical Corps, IDF, Beilinson Medical Center, Sackler School of Medicine, Tel-Aviv University, Petah-Tikva, Israel

Due to nature-induced or man-made disasters, mass casualties have become an integral part of modern life and are a common problem within the context of modern mass transportation systems, industry, and war technology. Toxicological disasters fall within this domain and similarly require detailed planning and exercise at the civilian, community, and hospital level. Industrialization has caused a major threat to the community with increased incidence of chemical disasters. Triage, or the sorting of casualties according to the severity of wounding and urgency of treatment, is the method applied in order to cope at the hospital level with large numbers of casualties that exceed the capacity of available teams to provide immediate medical care. To illustrate the application of these general principles in a disaster situation due to toxic agents, a hospital toxicological mass casualty schematic plan was designed for the management of organo-phosphorus (OP) poisoned victims. Casualties reaching the hospital are directed to a decontamination station where either a self-pressured shower is taken or it is provided by paramedical teams. So as not to impede the flow of casualties, only minimal treatment and life-saving procedures are given at this stage. After decontamination, a senior physician performs triage by grouping the casualties according to their need for assistance in respiration. Spontaneously breathing patients are regarded as mild or moderate casualties, while those needing respiratory support are treated by a resuscitation team that is expert in intensive care. The treatment scheme is based upon the fact that pharmacologic treatment by antidotes and respiratory support are the two main, life-saving modalities in the treatment of organo-phosphorus casualties. 


\section{Innovations, Airway and Ventilator Care \\ Chairman: Miroslav Klain \\ Moderator: David Nancekievill \\ Tuesday, 12 September, 14:00-15:30: \\ Theatre 2}

\section{S 3.3}

\section{The Laryngeal Mask Airway}

Archie IJ Brain

Newham General Hospital, London, UK

The Laryngeal Mask (L.M) is a new airway adjunct which, when inserted blindly into the pharynx, forms a low-pressure seal around the laryngeal inlet. Results of the author's experience in over 7000 surgical patients are presented. The device provides a clear airway which permits gentle positive pressure ventilation. The design avoids the dangers of lung barotrauma, laryngeal trauma, accidental one-lung occlusion, and esophageal misplacement. With simple training, it easily can be inserted in a few seconds in the majority of patients. Insertion does not require a laryngoscope or muscle relaxants and, provided the mouth can be opened and reflex responses are sufficiently obtunded, may be performed blindly in any patient position. Once inserted, it is exceptionally well-tolerated and normally will not be rejected until protective reflexes are restored. However, effective coughing can occur with the LM in situ, thereby reducing the need for suctioning. Once it is in this position, it is possible to intubate the trachea blindly through the lumen of the LM. Also, it is possible to insert a wide-bore tube into the oesophagus behind the positioned LM for gastric drainage without loss of seal. The LM does not prevent regurgitation and was developed for routine use in fasting patients undergoing general anesthesia. However, the speed and simplicity of insertion mean that hypoxia and upper airway obstruction can be corrected rapidly thereby reducing the risk of regurgitation at the outset. Experience in training paramedical personnel to use the LM suggests the necessary skill is acquired readily, but the importance of being able to recognise failure to achieve a clear airway should be stressed.

\section{S. 3.6}

Transtracheal High Frequency Jet Ventilation for Emergency Management during Cardiopulmonary-Cerebral Resuscitation (CPCR)

Miroslav Klain, E Benjaminsson, $R$ Levine, E Brader, P Safar International Resuscitation Research Center (IRRC) and Department of Anesthesiology and Critical Care Medicine, Montefiore Hospital, University of Pittsburgh, Pittsburgh, Pennsylvania, USA

Emergency airway management is the first step in successful CPCR. High frequency jet ventilation (HFJV) is capable of providing total respiratory support with a $14 \mathrm{G}$ catheter introduced by cricothyroid membrane puncture. It can obviate traumatizing intubation attempts in emergency situations. Because of the small size of the catheter, the danger of trauma to surrounding structures is much less than in emergency tracheostomy or even cricothyrotomy. Rapid control of the airway with immediate oxygen administration via the trachea facilitate the immediate establishment of effective ventilation, the prevention of hypoxemia, and diminution of cerebral insult. The safety of the method is increased further by monitoring airway pressure directly from the transtracheal cannula and by the use of cutoff should airway obstruction occur. Even under these circumstances, when ventilation is interrupted, sufficient oxygen is delivered into the trachea to prevent hypoxemia. As long as respiratory frequency is 100 breaths per minute or higher and the inspiratory duration is at least $30 \%$ of the cycle, aspiration does not occur. In addition, during external cardiac compressions, it is not necessary to interrupt compressions to administer mechanical breaths. Experience with cricothyroid membrane puncture and HFJV for emergency management of a difficult airway in 132 patients will be discussed. In summary, cricothyroid membrane puncture with high frequency jet ventilation or oxygen administration should be considered an important alternative for emergency airway management. Medicine.

* Supported in part by a grant from the Laerdal Foundation for Acute 


\section{S 4.2}

\section{Noninvasive Imaging in Cardiovascular Emergencies H Dirk Sostman \\ Duke University Medical Center, Durham, North Carolina, USA}

Established and emerging diagnostic imaging modalities have assumed paramount importance in the evaluation and management of cardiac and vascular emergencies. This presentation will describe the usefulness of computed tomography (CT), magnetic resonance imaging (MRI), ultrasound (US), and nuclear medicine techniques in emergency situations related to the cardiovascular system.

US is the most effective modality for the diagnosis of common heart conditions in the acute setting. These include cardiac tamponade, left ventricular outflow obstruction, endocarditis, cardiac masses and thrombi, and complications of myocardial infarction. For cases not suitably diagnosed by US, MRI may be of value in establishing the diagnosis of tamponade and particularly of mass lesions. The intrathoracic systemic vessels are, in general, best evaluated by CT and MRI. Aortic aneurysm and dissection are imaged best by MRI, but CT is accurate and more widely available. Similarly, obstruction of the great veins in the thorax is well-defined by both MRI and CT. The intra-abdominal vessels also are imaged most adequately using C'T and MRI, but in the abdomen, US also has reasonable diagnostic ability. There have been significant recent developments concerning the imaging diagnosis of deep venous thrombosis (DVT) and pulmonary embolism (PE). For DVT, noninvasive methods increasingly are replacing ascending phlebography for diagnosis and follow up. US is quick and accurate for the femoral and popliteal veins. MRI, while often less readily available, is equal to US in the the examination of the leg veins and is more accurate than MRI for detection of thrombosis above the inguinal ligament. There is new information concerning the scintigraphic diagnosis of PE from a multicenter American study. All of the above modalities must be considered against the reference standard of direct injection of intravascular contrast material for arteriography and venography. Examples of such correlations will be shown. Noninvasive imaging modalities increasingly are the most appropriate methods for diagnosis and triage of common cardiovascular conditions in the acute setting. Their safety, speed of acquisition, and the diagnostic information gained comprise a substantial recommendation for their widespread use.
S 4.3

Cardiovascular Assessment and Imaging of Myocardial Ischaemia

\author{
John M Low \\ Dept. of Anaesthesia and Intensive Care, Chinese University of \\ Hong Kong, Shatin, Hong Kong.
}

The myocardium is an organ very vulnerable to inadequate blood supply, and the consequences of myocardial ischemia can be diasastrous. Despite the use of expensive or highly technical monitoring equipment, intelligent interpretation of the data must be made in the light of clinical assessment.

1) Assessment of the circulation can be performed by numerous techniques, some more invasive than others. Blood pressure measurement by indirect and direct means, and continuous indirect (Penaz) methods now are available. Filling pressure, obtained by direct central venous cannulation and the use of flow-directed catheters has demonstrated the lack of correlation between right atrial and pulmonary capillary wedge pressures (PCWP). Myocardial ischemia results in reduced compliance, raised PCWP, and diminished cardiac output.

2) In assessing pump function, the major recent developments have occurred in the fields of ultrasound and radionuclide technology. Ultrasound techniques include the four major modes of $\mathrm{M}$ mode, 2D-mode, doppler echocardiography, and trans-esophageal echocardiography. Ejection fraction is the most useful clinical index of myocardial insufficiency. Many variants of radionuclide angiography have produced very sensitive means of detecting asymptomatic myocardial ischemia including use of single photon emission computerized tomography (SPECT) and positron emission tomography (PET). Conventional contrast angiography, used to define coronary obstruction, has been enhanced by the use of subtraction techniques, particularly digital subtraction angiography. 
Task Force 1.2

Wednesday, 13 September 09:00-10:30: Symposium 301

\section{TF 1.2.2}

International Medical Cooperation by the Japanese Government in Disaster Relief and the Future of this Program

Yasuhiro Yamamoto

Department of Emergency and Critical Care Medicine, Nippon Medical School, Tokyo, Japan

Ten years ago, the Japanese government initiated a donor program of international medical cooperation with the establishment of the Japanese Medical Team for Disaster Relief (JMTDR). From the outset, this team has operated only on request from disaster-stricken countries. It has experienced problems with alerting systems which frequently have resulted in mobilization of this rapid deployment team too late to be effective in sudden impact disasters. JMTDR was mobilized first during the African drought of 1984-85, when a team of 32 staff was deployed to Ethiopia for four months. They provided care to 1,100 patients and supplied 16,000 $\mathrm{kg}$ of medical goods. In order to obviate any imbalance between the differing emergency care facilities which results in unequal distribution of victims, these goods deliberately were disbursed on a simple scale, correlating with the basic equipment and drug allotment of other teams. Also, JMTDR was deployed to the September 1985 earthquake in Mexico, D.F. It arrived just 39 hours after the seismic event. While other teams were well staffed and medically equipped, the Japanese team provided special search and rescue equipment in the form of fiber-optic scopes which proved valuable in victim-search operations. These experiences have taught the value of detailed briefing information in deciding the composition of and equipment for the team in order to meet the specific needs of each individual disaster. It is of paramount value that such Relief Teams have expert information on: 1) The indigenous resources and the system of health care in recipient countries; 2) The political, consular, administrative, and logistic protocols for delivery of assistance; 3) The cultural environment in recipient countries; and 4) The specific needs of each incident. JMTDR now numbers over three hundred members and represents a rapid deployment facility with unique capabilities. In collaboration with Japanese non-governmental organizations (NGOs) and established international agencies, the JMTDR will look to play an increasing role in the provision of humanitarian and material aid for victims of disasters worldwide.
Symposium Session 5

Anaesthesia and Pain Control

Chairman: Wolfgang Dick

Moderator: Peter Baskett

Wednesday, 13 September, 09:00-10:30: Convention Hall

\section{S 5.3}

The Role of the Combitube for Difficult Intubations in Emergency Situations

Michael Frass, B Watschinger, A Podolsky, C Leithner

2nd Department of Internal Medicine, University of Vienna, Vienna, Austria

Under certain circumstances, emergency intubation with the conventional endotracheal airway (ETA) may be difficult. A new airway, the esophageal tracheal combitube (Combitube ${ }^{\mathrm{TM}}$, Sheridan Catheter Corp., Argyle, NY) allows effective ventilation independent of esophageal or tracheal placement. The Combitube has been used successfully in eight cases of difficult intubation during cardiopulmonary resuscitation (CPR). On three occasions, the Combitube has been used during CPR in the general ward due to inadequate access to the patient's head; the Combitube was inserted three times because of failure of endotracheal intubation caused by the presence of bull-neck. In two patients, visualization of the vocal cords was impeded, once due to massive regurgitation and once due to bilateral compression of the pharynx caused by hemorrhage from the carotid artery after unsuccessful attempts to puncture the internal jugular vein. Patients have been ventilated through the Combitube in the esophageal position for up to eight hours. Blood gas values were comparable to those obtained during subsequent ventilation through a conventional ETA. The Combitube might prove a valuable tool for difficult intubations in emergency situations. The advantages, in comparison to the ETA, are quick insertion, effective ventilation in esophageal or tracheal position, fixation of the tube by the pharyngeal balloon, and minimal cervical spine movement. 


\author{
Symposium Session 7 \\ Trauma, Shock \\ Chairman: Kimball Maull \\ Moderator: Konrad Messmer \\ Wednesday, 13 September, 09:00-10:30: Theatre 1
}

S 5.4

Emergency Airway Control in Trauma Patients

Charles Barton, I Stene, Chris Grande, B Burns

Department of Anesthesia, R Adams Cowley Shock-Trauma

Center, MIEMSS, University of Maryland Medical Systems,

Baltimore, Maryland, USA

Initial airway management in patients who have sustained severe trauma provides a challenge for clinicians. Adequate oxygenation can significantly reduce the risk of hypoxic cardiac arrest, CNS damage, and subsequent organ failure. Rapid tracheal intubation frequently is required early in the resuscitation process as well as prior to surgical intervention. Because of its rapid onset, succinylcholine $(\mathrm{SCH})$ has been the neuromuscular blocking agent (NMBA) of choice. SCH has several undesirable effects which include increased intragastric, intraocular, and intracranial pressures, and hyperkalemia in burn and spinal cord injured patients (PTS). In this study, we used high-dose vecuronium (VEC, $0.28 \mathrm{mg}$ / $\mathrm{kg}$, i.v.) in place of SCH. VEC is a NMBA with none of the adverse effects of SCH.

\section{METHODS:}

After institutional approval, twenty-five trauma patients were studied. Monitoring included ECG, blood pressure (BP), pulse oximetry, temperature, and mass spectrometry. The electromyogram (EMG) from the adductor pollicis was recorded while a train of 4 (T4) stimulations was delivered to the ulnar nerve every 10 seconds. PTS were intubated after the T-1 of the T- 4 was depressed to $90 \%$ of baseline.

\section{RESULTS:}

$90 \%$ neuromuscular blockade (NMB) occurred at $77.4 \pm 4.2 \mathrm{sec}$ (sem). The duration of NMB to $25 \%$ recovery of $\mathrm{T}-1$ was $88.8 \pm 5.3$ minutes. Intubating conditions were excellent. No side effects of VEC were observed. Heart rate and BP remained stable.

\section{DISCUSSION:}

VEC is a safe alternative NMBA for emergency airway control in PTS at risk for side effects from SCH. VEC, $0.28 \mathrm{mg} / \mathrm{kg}$, provided rapid relaxation for intubation and prolonged $\mathrm{NMB}$ with no adverse effects.

\section{S 7.2}

\section{Rapid Infusion Catheters}

$S$ Stevens and Kimball Maull

Department of Surgery, University of Tennessee Medical

Center, Knoxville, Tennessee, USA

Rapid fluid administration is the cornerstone of successful trauma resuscitation. Percutaneous insertion of catheter introducers has gained wide acceptance as a quick and reliable means of rapid intravascular volume expansion. Factors that affect rapid fluid resuscitation with these devices include kinking of catheter introducer, the type and temperature of infusate, and diameter of coapted administration tubing. Rates of flow through 8.5 French (Fr.) catheters from zero to $80^{\circ}$ of catheter angulation were determined in vitro for various fluids (crystalloid, whole blood, diluted packed cells) and administration tubing of different sizes (regular IV tubing, blood tubing, and large-bore trauma tubing). The flow rate of crystalloid infusion through blood tubing was found to be approximately double that of regular IV tubing $(316 \mathrm{ml} / \mathrm{min}$ vs 160 $\mathrm{ml} / \mathrm{min}$.), and trauma tubing had approximately three times the flow rate of blood tubing $(805 \mathrm{ml} / \mathrm{min}$.). Warmed, diluted packed cells could be infused almost twice as fast as cold whole blood (642 $\mathrm{ml} / \mathrm{min}$ vs $340 \mathrm{ml} / \mathrm{min}$.). Kinking of the catheter introducer, a heretofore poorly described phenomenon, halved the flow rate of fluids through large-bore trauma tubing $(805 \mathrm{ml} / \mathrm{min}$ vs $350 \mathrm{ml} /$ min.) but had no effect when standard IV tubing was utilized. Piggybacking blood into an existing IV line instead of infusing it directly into the catheter can decrease blood flow $94 \%(340 \mathrm{ml} /$ min vs $20 \mathrm{ml} / \mathrm{min}$ ). It is concluded that a large-bore catheter, by itself, does not guarantee high flow rates. Physician recognition of these concepts can result in improved resuscitation of hypovolemic patients. 
S 7.4

\section{The Trauma Patient Presenting with Hypotension and Euvolemia

\author{
Department of Surgery and Intensive Care, St. Joseph's
} \\ Peter $R$ Knight and PA Powles Hospital, Hamilton, Ontario, Canada}

This presentation will consider the pathophysiology of hypotension in the presence of an euvolemic state following trauma. Physiological principles will be used to aid in the approach to assessing the traumatized patient with this puzzling diagnostic challenge. The discussion will include the possible role of central nervous system injuries and conditions, the role of cardiac pump failure from different aetiological causes, and possible pathological processes involving the respiratory system which could be involved. Endocrine system abnormalities also will be considered, together with the role of numerous medications which may have been used by the patient will be discussed. Finally, a practical approach to a patient who has been involved in trauma of varying severity who presents in the emergency room with hypotension will be developed and presented.

\section{S 7.5}

\author{
Haemodynamic Aspects of Septic Shock \\ LG Thijs \\ Medical Intensive Care Unit \\ Free University Hospital, Amsterdam, Netherlands
}

The incidence of sepsis still is rising. In $25-40 \%$ of the cases, the syndrome, septic shock ensues which has a mortality rate of about $50 \%$. It is increasingly recognized that host responses (TNF, IL-1, activated complement factors, etc.) rather than the microorganisms or their products are responsible for the syndrome. The most striking features of septic shock are, at least initially, complex alterations in micro and macrocirculation. These may be responsible for progressive organ failure, the cause of death in many cases. The first hemodynamic change observed at the bedside is a decrease in systemic vascular resistance with a reflex increase in cardiac output. Apart from vasodilation, redistribution of blood flow and of blood volume ("pooling") are important features. In the microcirculation, at least three mechanisms seem to be operative: vasodilation; microembolization (granulocytes, platelets); and endothelial cell injury with cell swelling and increased permeability of the microvasculature. These mechanisms interfere with the finely tuned autoregulatory processes that normally adapt blood flow and oxygen transport to tissue oxygen needs. The resulting increased inhomogeneity and maldistribution of flow is thought to be responsible for the ineffective oxygen uptake by the tissues, which is a fundamental defect in septic shock. These abnormalities also may be the pathophysiologic basis of the observed abnormal supply dependency of oxygen consumption in septic shock. In many patients, persistent vasodilation is observed (peripheral vascular failure) which seems to contribute to an unfavourable outcome. Decreased myocardial competence with resulting decline in cardiac output is well-documented in septic shock. However, also during the hyperdynamic phase, myocardial depression has been observed possibly related to the presence of a myocardial depressant substance and presumably not related to decreased coronary flow. In the presence of pulmonary hypertension, the right heart may dilate. This increases the need for oxygen and interferes with the oxygen supply/demand balance of the right heart. In some cases, right heart failure accounts for a decline in cardiac output and significantly contributes to mortality. Understanding of the hemodynamic alterations in an individual patient forms the basis of appropriate hemodynamic supportive treatment. 
Plenary Session 02

Trauma: Maxillofacial

Chairman: Sabri Shuker

Moderator: Hugh Cannell

Wednesday, 13 September, 11:00-12:30: Convention Hall

P 2.5

Pathophysiological Effects of Blast on Maxillofacial

Region

Sabri T Shuker

Maxillofacial Unit, Karkh Emergency Hospital, Baghdad, Iraq

Maxillofacial blast injuries are becoming increasingly more common in contemporary warfare. More rockets are being used and they are destined to dominate future battles. These sophisticated weapons, with their immense explosive potential, result in casualties which may be too numerous even for experienced medical personnel to handle. Our experience in the recent Iraq-Iran war has shown that facial bone fractures due to blast are of a wholly different pathology than fractures seen in civilian trauma or penetrating shell fragment injuries. Often, their management is complicated by associated injuries to the eyes, brain, lungs, etc. The most commonly observed maxillofacial injuries in victims who survived rocket blast consisted of soft tissue shredding, burns with shredding, transverse mandibular fractures, and severe crushed-eggshell fractures of the middle facial bones with or without associated penetrating missile injuries. Consideration of the biodynamics of very high energy, blast wave, frontal injuries presented in this paper, permits the assessment of injuries in the context of such explosions and contributes to the rationale of management. Such considerations may, in addition, further assist in the development of shelters and other protective devices.
Plenary Session 03

Blood Supplies, Conservation

Chairman: Anis Baraka

Moderator: Mattias Kalina

Wednesday, 13 September, 11:00-12:30: Theatre 2

\section{P 3.6}

Experience of a New Rapid Infusion System for Blood Replacement during Liver Transplantation, Major Vascular, and Trauma Surgery

Michael F Smith, G Collins, JR Klinck, MJ Lindop

Department of Anaesthesia and Intensive Care, Addenbrooke's Hospital, Cambridge, UK

The Haemonetics "Rapid Infusion System" (RIS) was used clinically during liver transplantation, and major vascular and trauma surgery between April 1988 and May 1989. The RIS is a portable transfusion system which has the capability of rapidly transfusing homologous/autologous blood at high flow rates and at physiological temperatures. The RIS has the capability to infuse blood at rates of up to $1500 \mathrm{ml} / \mathrm{min}$ at a temperature of $39^{\circ} \mathrm{C}$. The RIS has been used during 35 operative procedures and the results have been encouraging. There have been 5 occasions when massive blood loss has occurred unexpectedly and the ability to assemble the RIS for use in under 3 minutes is very impressive. The temperature homeostasis of the patients has been improved and in some cases of really massive loss, the RIS acts as a heat source for the patients. Normally, such patients would be at risk of becoming rapidly hypothermic. One patient died of uncontrollable hemorrhage after a blood loss exceeding 85 liters, yet the esophageal temperature at the time of death was $37.5^{\circ} \mathrm{C}$. Eight other patients had blood losses from 40 liters to greater than 120 liters; all survived surgery and were normothermic on transfer to the ICU. The use of the RIS in conjunction with the Cell Saver has proven to be an effective means of rapidly processing and returning red cells to the patient.

\begin{tabular}{|c|c|c|c|c|}
\hline & \multicolumn{4}{|c|}{ RAPID INFUSION SYSTEM RESULTSFOR 35 CASES } \\
\hline & $\underline{\text { Loss }}$ & Reinfused & Bank blood (u) & Cell Saver \\
\hline Mean (L) & 25.7 & 28.9 & 10.6 & 12.9 \\
\hline Range (L) & $2.8-120.0$ & $3.8-129.0$ & $4-100$ & $0.5-90.0$ \\
\hline
\end{tabular}

Blood losses of $30-40 \mathrm{ml} /$ minute are the lower level at which the RIS begins to become efficient. For routine low blood loss surgery, the use of the RIS can prove counterproductive because of the large dead space of the RIS circuit. 


\section{P 4.1}

Prehospital Trial of Tissue Plasminogen Activator (t-PA): Paramedic Education and Training W Brian Gibler

Vanderbilt University Medical Center, Nashville, Tennessee, USA

The paramedic education and training program for the prehospital delivery of t-PA (Genentech, Inc.) in Nashville required the separation of various aspects of patient evaluation and drug delivery into discrete modules. This program required 24 hours of classroom instruction for each of the 31 paramedics who completed the course. To supplement verbal instruction, a 30-minute, paramedic training video was produced. Paramedics received a comprehensive 3-ring binder detailing and supplementing all the information given during the course.

Initially, an intensive overview of the pathogenesis of acute myocardial infarction (AMI), thrombogenesis, and thrombolysis was delivered in lecture format. After this instruction, the presentation of methods of patient evaluation for acute myocardial infarction involved the development of a specific history, cardiac-oriented, physical examination, and inclusion criteria format suitable for field use. Each of these areas received individual emphasis, through lecture presentations followed by paramedic role-playing. The prehospital diagnosis of cerebrovascular disease and dissecting aortic aneurysm were stressed. Following this introduction, cardiacoriented physical examination and exclusion criteria were combined and practiced in role-playing sessions. The verbatim reading of the prehospital informed consent was practiced by the paramedic after a patient without an exclusion criterion was found to have an AMI.

Finally, the Marquette MAC PCTM 12-lead electrocardiogram/ cellular phone unit along with the protocol for correct electrode placement for performing a 12-lead electrocardiogram was presented to the paramedics. To complete the manual skills portion of this program, instruction was given for the mixing of lypholized t-PA and loading it into the Pharmacia Deltec CADD-t-PA ${ }^{\mathrm{TM}}$ constant infusion pump. At the conclusion of the 24-hour session, each paramedic was required to demonstrate the successful completion of every aspect of the course, including a written examination. This paramedic education and training program has been designed to be duplicated on a broad scale should prehospital delivery of thrombolytic agents prove feasible and safe.
P 4.3

Clinical Evaluation of Prehospital Thrombolysis with APSAC in Acute Myocardial Infarction: Preliminary Report

\author{
Pierre Mols, N Picart, O Polis, G Fautsch, Arsene Mullie, $R$ \\ Bernard \\ Belgian Eminase Prehospital Study Group, Brussels, Belgium
}

\section{AIM:}

To evaluate the feasibility, safety, and clinical outcome of prehospital thrombolysis (PT) in patients with acute myocardial infarction (AMI) using APSAC (Eminase R) as a fibrinolytic agent.

\section{METHODS:}

Six hospitals respectively located in Antwerp, Brugge, Brussels, Charleroi, Gand, and Namur participated in the study. Three of them had available a suburban emergency medical services (EMS) system and the others an urban system. Criteria for inclusion in this study group were: a) typical thoracic pain that appeared at least 30 minutes before but within the last 4 hours; b) ST-T segment elevation >2mm in at least two ECG leads; c) absence of clinical and ECG improvement after nitrates; and d) oral informed consent from the patient or a family member. Exclusion criteria were those classically accepted when fibrinolysis is performed. Treatment consisted of usual management of the patient associated with the IV injection of 30 units of APSAC in 5 minutes or as soon as possible after the arrival of the ambulance. Time gain was the time interval between time of arrival ambulance at the scene plus the time of arrival at emergency department. Time to CPK peak, CPK peak value, clinical reperfusion, and coronary patency were evaluated.

\section{RESULTS:}

42 consecutive patients (pts) were included in the protocol during the first 6 months of the study. There were 38 men and 4 women. The mean age was $59 \pm 9$ years (Mean $\pm 1 S D$ ). An inferior wall AMI was present in 24 patients and 17 had anterior wall infarctions. One patient with acute pericarditis erroneously was selected $(2.4 \%)$. The mean time from onset of the pain to fibrinolysis was $89 \pm 43 \mathrm{~min}$ and the mean time gain was $50 \pm 3$ minutes (range $26-120 \mathrm{~min}$ ). The time gain was the same in urban and suburban EMS systems (54 520 min versus $45 \pm 10 \mathrm{~min}$ ). Using clinical criteria, reperfusion was observed in $50 \%$ of the patients. CPK peak of $<12$ hours was observed in $61 \%$ of the pts, and coronary arterial patency was confirmed in $83 \%$. Time gain, angiographic patency, CPK peak and time to CPK peak were not statistically different between patients with successful or unsuccessful reperfusion. In the prehospital period, three patients developed ventricular fibrillation, and one cardiogenic shock. During hospitalization, severe bleeding occurred in one patient, and acute pericarditis in 2. Two patients died ( 1 from cardiogenic shock, and 1 from prolonged bleeding and multiple organ failure).

\section{CONCLUSION:}

In Belgium, prehospital thrombolysis seems to be feasible and safe. The time gain is estimated at about $50 \mathrm{~min}$ for both urban and suburban EMS systems. The benefits of PT, in terms of mortality and morbidity, still remain to be defined by a multicentric, controlled, clinical study. 
WAEDM, Task Force 3

Aircraft Incidents and Aeromedicine

Chairman: Morgn Fahey

Task Force 3.3

Thursday, 14 September, 9:00-10:30: Symposium 301

\section{TF 3.3.2}

The NLDB Aircraft Accident Study the Injuries and Consequences of the M1 Aircraft Accident

\section{A New Thrombolytic Regimen for Acute Mycardial Infarction Using Combination Half Dose Tissue Plasmino- gen Activator with Full Dose Streptokinase \\ Cindy L Grines \\ Cardiology Division, University of Kentucky Medical Center, Lexington, Kentucky, USA}

Since a previous study utilizing a combination of tissue plasminogen activator ( $t-P A$ ) and urokinase demonstrated reduced reocclusion rates compared to t-PA alone, this study was conducted to determine whether the combination of t-PA and streptokinase might achieve similar results at reduced cost. Forty patients with acute myocardial infarction were treated with a one hour infusion of t-PA ( $50 \mathrm{mg})$ and streptokinase ( 1.5 million units) administered within six hours of symptom onset (mean=3.6 11.2 hours). Emergency coronary arteriography revealed patency of the infarctrelated artery in $30 / 40(75 \%)$ of the patients. With the addition of coronary angioplasty in those who failed thrombolytic reperfusion, acute patency was increased to $98 \%$. In-hospital mortality $(2.5 \%)$ and the incidence of significant bleeding requiring transfusion $(15 \%)$ was low. Angiographically documented reocclusion of the infarct vessel occurred in $3 / 37(8 \%)$ patients by day seven. Regional wall motion of the infarct zone improved by $0.9 \pm 0.9$ standard deviations / chord ( $p<.0005)$, and ejection fraction increased $3.6 \pm 8 \%$ $(p<.05)$ between acute and day 7 studies. Compared to the price of full dose t-PA $(\$ 2,300)$ or t-PA with urokinase $(\$ 3,500)$, the cost of this regimen was $\$ 1,230$ per patient. This pilot study demonstrates that at half the cost, a combination of half dose t-PA with full dose streptokinase offers high infarct vessel patency, recovery of ventricular function, a low rate of reocclusion, and few bleeding complications. To assess the precise comparability of our combined regimen to other thrombolytic approaches will require a large, randomized, prospective trial which is ongoing.

\author{
John $M$ Rowles, G Kirsh, DJA Learmonth, JP Martindale, and \\ the Nottingham, Leicester, Derby, Belfast Group \\ The Department of Orthopaedic and Accident Surgery, \\ University of Nottingham, Queen's Medical Centre, Notting \\ ham, England
}

On Sunday, 8 January 1989, a Boeing 737 bound from Heathrow to Belfast crashed while attempting an emergency landing at East Midlands Airport. It crashed onto the western embankment of the Ml motorway, one of the busiest in the country. One hundred twenty-six passengers and crew were on board. Eighty-seven passengers and crew survived the impact and were transferred to one of four hospitals in the region. This paper reviews the factors that influenced the ability of primary hospitals to cope with the disaster. These include: a) Time; b) Geography; c) Communications; and d) Resources. Four patients died soon after arrival and 201 fractures have been identified among the 83 survivors. Patterns of injuries have emerged and these are being investigated as to the mechanisms of injury. The workload created as a consequence of the sudden intake of 87 multiply injured patients has been reviewed. The NLDB Study Group was established within three days of the crash and, as a result, important lessons have been learned with respect to disaster management. 
Symposium Session 10

Education, Undergraduate

Chairman: Louis Binder

Moderator: Joe Epstein

Thursday, 14 September, 09:00-10:30: Theatre 2

\section{S 10.1}

Undergraduate Education in Emergency Medicine Louis S Binder

Director Division of Emergency Medicine, Texas Tech University Health Sciences Center, El Paso, Texas, USA

The recognition and management of common processes which present in acute care and the acquisition of resuscitation expertise are important skills for all physicians. Primarily for this reason, emergency medicine content should be a required part of the general education of the physician. Emergency medicine has much to offer the undergraduate medical curriculum. The emergency department potentially is a major source of ambulatory patient visits, assignment to the emergency department provides students with exposure to a diversity of unscreened patients, access to acutely ill patients requiring intervention, and promotes the development of life-saving and professional time management skills. However, thus far, emergency medicine has failed to gain acceptance and integration into the traditional medical school curriculum. Persistent efforts by worldwide faculty and development of effective, concise, and easily implemented curricula in emergency medicine are needed to accomplish this objective. Effective approaches for advancing undergraduate emergency medicine education will require: 1) The delineation of unique aspects of emergency medicine which would be of benefit in the undergraduate curriculum; 2) Definition of which of the aspects of emergency medicine should be taught in the basic sciences and clinical curriculum; and 3) Definition of the educational goals that are crucial for the advancement of emergency medicine education. In addition, we must strive for: department status; liaison with academic/governmental/professional societies which regulate curriculum content; integration into medical school administration and existing educational committee structures; and 4) Effective identification and recruitment of good future emergency physicians beginning at the medical student level.

\section{Plenary Session 06 \\ Innovations}

ACLS Chairman: Tom Evans

Moderator: Nicholas Bircher

Thursday, 14 September, 11:00-12:30: Convention Hall

\section{P 6.2}

\section{High Dose Epinephrine and Coronary Perfusion During Cardiac Arrest in Humans}

Norman A Paradis, GB Martin, EP Rivers, J Rosenberg, TJ Appleton, Richard M Nowak

Department of Emergency Medicine, Henry Ford Hospital, Detroit, Michigan, USA

Work in animal models of cardiac arrest indicates that the optimal dose of epinephrine (EPI) used in resuscitation efforts may be larger than that used clinically. The aortic (Ao) to right atrial (RA) pressure gradient (Ao-RA) during CPR relaxation phase is the coronary perfusion pressure in the arrested heart, and is positively correlated with return of spontaneous circulation (ROSC).

The study population consisted of adults in medical cardiopulmonary arrest who had failed to respond to prolonged standard ACLS therapy. Aortic arch and RA catheters were placed, and simultaneous pressures were recorded. The maximum rise in AoRA after a standard $1 \mathrm{mg}$ dose of EPI (SD) and a high dose (HD of $0.2 \mathrm{mg} / \mathrm{kg}$, i.e. $14 \mathrm{mg}$ in a $70 \mathrm{~kg}$ patient) were measured during the 5 minutes following IV administration. The protocol was sequential in that each patient served as his/her own control by receiving $\mathrm{SD}$ first followed by HD.

Mean age was $59 \pm 16$ years. Time from arrival to $H D$ was $25 \pm 8$ minutes. Pressures are in $\mathrm{mm} \mathrm{Hg}$ for patients in which catheter placement was confirmed radiologically $(\mathrm{N}=32)$. Baseline Ao-RA was $4.9 \pm 6.6$ before $\mathrm{SD}$, and $5.6 \pm 8.4$ before $\mathrm{HD}$ (not statistically different, $p=0.54$, Students t-test paired samples). The mean maximal increase in these gradients was $3.4 \pm 5.0$ (range: 2 to 12 ) after $\mathrm{SD}$ and $11.8 \pm 10.7$ (range 0 to 45 ) after the $\mathrm{HD}(\mathrm{p}=0.001)$. Four patients had ROSC after receiving HD therapy. High dose EPI significantly increases the Ao-RA gradient during CPR in humans, which may improve myocardial blood flow and rates of ROSC. 
P 6.3

Controlled Clinical Trials of Graded Epinephrine Doses
for Cardiac Arest
PaulE Pepe, CG Brown, JP Ornato
Baylor College of Medicine, Houston, Texas, Ohio State
University, Columbus, Ohio and the Medical College of
Virginia, Richmond, Virginia, USA

Recent experimental evidence suggests that, despite basic CPR and $0.02 \mathrm{mg} / \mathrm{kg}$ of intravenous epinephrine (EPI) every 5 minutes (normally very potent doses of EPI during spontaneous circulation), most cardiac arrest patients remain in profound circulatory collapse, and, as a result, their aortic diastolic pressure remains too low to adequately reperfuse the heart. The current recommended dose of EPI for adults in cardiac arrest (i.e., $1.0 \mathrm{mg}$ or about 0.02 $\mathrm{mg} / \mathrm{kg}$ ) has been demonstrated in animal models, to be too low. Both experimental and clinical data suggest that a 10 -fold increase in dose $(0.2 \mathrm{mg} / \mathrm{kg})$ may be more appropriate after 10 minutes of CPR. As time passes during CPR, a progressive tachyphylaxis or uncoupling phenomenon seems to occur in the vascular alpha receptors, resulting in the need for progressively higher doses of EPI. The purpose of this report is to review the rationale, methodologies, preliminary observations, and clinical caveats regarding a multi-center, randomized, controlled study-in-progress: the purpose of this study is to compare the effects of blinded doses of 0.02 vs $0.20 \mathrm{mg} / \mathrm{kg}$ EPI in patients remaining pulseless following initial, standard management of out-of-hospital, unmonitored, cardiac arrest. Currently, it appears that multiple factors-such as duration of arrest, time to basic CPR, body weight, and individual variability all seem to play a significant role in determining the appropriate EPI dose. Potential adverse complications of higher EPI doses still need to be defined.

\section{Plenary Session 07 \\ Education, Postgraduate \\ Chairman: Richard Nowak \\ Moderator: Herman DeLooz \\ Thursday, 14 September, 11:00-12:30: Theatre 2}

P 7.1

Emergency Postgraduate Training-United States
Richard M Nowak
Chairman Department of Emergency Medicine, Henry Ford
Hospital, Detroit, Michigan, USA

Currently, there are 75 Emergency Medicine residencies approved by the Residency Review Committee/Emergency Medicine (RRC/ EM) in the United States. These programs graduate approximately 450 Emergency Medicine specialists each year. Currently, there are 1,404 residents in training in RRC/EM-approved programs. Thus far, 2,843 residents have graduated from these residencies. At the present time, the minimum training period for approved programs is three years. However, there are a number of residencies that require a full four years of postgraduate training. Programs are comprised either of a three or four year format. At the present time, there are no approved dual track, single residency training formats. There is debate concerning the optimal period for training. Many suggest that a fourth year is necessary for those who wish to pursue an academic/research career. The format for this fourth year of training has not been established and programs that are providing the four year format still are attempting to develop an optimal curriculum. Whether this trend towards four year programs will continue to be used for the development of academic physicians will be explored. The pros and cons of a fourth year of postgraduate training will be presented. There are a variety of Emergency Medicine fellowships available for those completing postgraduate training in Emergency Medicine. At last count, there were 38 such fellowship programs. Training includes areas such as: basic emergency medicine research; toxicology; medical decision-making; emergency medical services; clinical research and teaching; pediatric emergency medicine; medical education; health policy administration in emergency medicine; and administration. The issues of who should accredit these fellowships, what should comprise that accreditation, and what the relationship of these fellowship programs should be to the current four year training format will be discussed. 


\section{P 7.2}

\section{The Development of Postgraduate Training Programs in Emergency Medicine in the United Kingdom} David $W$ Yates

Director, Accident and Emergency Services, Hope Hospital, Manchester, University of Manchester, UK

In recent years, many new medical specialties have developed in response to scientific and technical advances. In contrast, Emergency Medicine in the UK developed as a response to professional and consumer pressure. Early in the $1970 \mathrm{~s}$, it was expedient politically to "create" the specialty. This was remarkably successful in terms of department management, but the lack of a scientific base made it very difficult to formulate a coherent system of academic training. The latter was achieved just over 10 years ago by the establishment of a handful of Higher Specialist Training Programs under the control of a Specialist Advisory Committee (SAC). This is the usual format for all specialist training in the UK, each SAC being responsible to the appropriate Royal College. The catholic interests of Emergency Medicine have been addressed by a unique relationship of the SAC with both the Royal College of Surgeons and the Royal College of Physicians. This arrangement has been very effective. The number of training programs has grown steadily and there are now $\mathbf{5 5}$ Senior Registrars in post, with an expansion to 63 planned over the next 12 months. The four year program is available to those graduates who already have undertaken General Professional Training-usually for between 5 and 7 years after leaving Medical School. It consists of obligatory training in Accident and Emergency Departments and specialties which complement the candidates previous experience. Paediatrics, Intensive Care, Coronary Care, Medicine, and Surgery are mandatory. Further exposure for a wide selection of other specialties is recommended. All programs are inspected regularly by the SAC. Withdrawal of recognition is not uncommon and, in recent years, has led to a great improvement in the quality of training. Presently, the postgraduate examination system is under review. A specialist diploma is recognized increasingly as being appropriate and faculty status within one of the Royal Colleges is being pursued. Widespread support within and without the speciality for these developments should ensure the future of Emergency Medicine in the United Kingdom.

\section{P 7.4}

Diploma in Immediate Medical Care

I Heywood, Andrew K Marsden, B Steggles

Royal College of Surgeons, Edinburgh, Scotland

The Royal College of Surgeons of Edinburgh has developed and established a postgraduate medical diploma in (prehospital) emergency care. Titled: "The Diploma in Immediate Medical Care"(DIMC RCSEd), the examination tests the application of life support skills, theoretical knowledge, and practical ability of the practitioner in the field. This is believed to be the first examination of its type. The examination is open to fully registered medical practitioners who actively are involved in pre-hospital emergency care. It is suitable for "Immediate Care" doctors who practice roadside resuscitation, acute cardiac care, or sports medicine. It is valuable particularly for doctors in the armed forces. Candidates should have spent six months working full time in an Accident and Emergency Department or demonstrate equivalent experience. A full syllabus is available from the College and a reading list has been prepared by BASICS. The theory paper consists of two parts: a 20 question, single answer, multiple choice questionnaire linked with a 10 question, cardiac arrhythmia recognition quiz; and a six question, "short notes" paper testing knowledge of drugs, therapeutic principles, equipment, and/or the approach to common clinical emergencies. There is a triage exercise which tests the immediate organization, management, and prioritization of a number of casualties. In addition, there are three practical examinations: The "core skills" station tests the candidate's ability to maintain an airway and perform cardiopulmonary resuscitation on adult and paediatric manikins and to use essential pieces of emergency equipment including the defibrillator, oxygen therapy apparatus, and pain-relieving gas mixtures. The medical station examines the candidate's practical approach to myocardial infarction, acute asthma, drowning, hypothermia etc., and tests ECG and arrhythmia familiarity. In the trauma station, the candidate is led through a scenario involving one or two skillfully moulaged injured casualties in a field setting. Examiners are drawn from practitioners in prehospital care with backgrounds in general practice, anesthetics, accident and emergency medicine, cardiology, neurosurgery, pediatrics, etc. Successful candidates are received into associate membership of the Royal College of Surgeons of Edinburgh. Our presentation relates the experience and results of the initial five diets of the examination over its first year. 


\section{Symposium Session 12 \\ Strategy, Research \\ Chairman: William Spivey \\ Moderator: Garry Phillips \\ Thursday, 14 September, 14:00-15:30: Theatre 2}

\section{S 12.3}

\section{Research in Emergency Medicine-Canada: The Third Revolution}

\author{
Bruce MT Rowat \\ Professor, University of Toronto, Toronto, Ontario, Canada; \\ Emergency Department, Toronto General Hospital
}

Having addressed issues of exemplary patient care and quality postgraduate education in emergency medicine-research, the third revolution, is the challenge facing emergency medicine in the decade ahead. It may pose the most difficult barrier to face emergency medicine. Where there is no research, the specialty perishes (Proverbs 29:18). The survival of emergency medicine as an academic entity is contingent on the development of its own research base. The breadth of emergency medicine in and of itself poses certain difficulties; when research is more and more about less and less, a focused approach can yield a greater economy of effort and return on investment. In some instances, this has led to collaborative research activities in emergency medicine between traditional specialties. This may or may not be helpful, strategically speaking, for the development of either emergency medicine, or a personal career in research. Prehospital care and the investigation and treatment of acute medical and surgical conditions are areas of active research in Canada at this time. Issues of quality of working life in emergency medicine also are being examined. An overview of the current research activities in emergency medicine in Canada will be provided.

\section{S 12.4}

Research in Emergency Medicine-Japan
Takashi Ukai
Vice Director, Senri Critical Care Medical Center, Osaka,
Japan

Prior to the establishment of the Japanese Association for Acute Medicine (JAAM), research in emergency medicine in Japan was no more than sporadic. Even though they were accepted in the meetings of the Japan Surgical Society or other medical associations, papers on emergency medicine, if any, were offered only low profile sessions. JAAM, starting with about one thousand members in 1973, has grown rapidly and has developed into one of the largest medical associations in our country. It has 6,550 doctor members and more than 500 free papers are submitted for presentation at its annual meetings. Several local branch associations and affiliated medical associations such as the Japanese Association of Clinical Toxicology and the Japanese Association for the Surgery of Trauma, etc., were established. A nurse section and an ambulance personnel section of JAAM also have been established and hold regular, nationwide general meetings and local branch meetings. Initially, research in emergency medicine in JAAM was directed mainly at hemorrhagic shock, the pathophysiology of multiple trauma and burn injury, shock lung, ARDS, etc. However, in recent years, emphasis gradually has changed to that of DOA and CPR, DIC, sepsis, AMI, and CVA. Although papers on acute poisoning initially were rare, their numbers have increased substantially. This has resulted in the establishment of the new medical association mentioned above. In recent years, reports on multiple organ failure (MOF), chemical mediators of organ failure, and severity indices of organ failure have become the focus of attention. Among these, arterial blood ketone body ratio (AcAc/B-HOB) and reflecting redox potential of liver mitochondria have emerged as a prospective prognostic index of organ failure. Development of new therapeutic measures such as transarterial embolization, fiber-optic hemostasis of upper gastro-intestinal bleeding, organ support devices such as hemopurification systems, auxiliary artificial heart, and ECLA have emerged as topics of research. Recent attention has focused on mental health care of both emergency patients and personnel working in the stressful emergency medical environments, and on medico-ethical problems in emergency health care. Studies on EMS systems and statistical analysis of emergency patients constantly are being reported from diverse institutions. In the near future, advances are expected in the utilization of the so-called new media in the field of emergency medical services systems. Personal health data, including imaging (X-ray, CT, NMR, ultrasonic image, etc.), easily may be transmitted using cellular telephone systems or satellites and an enormous amount of personal health data may be printed in a small optical card to be used at OPDs or emergency rooms. 
Symposium Session 16

Disaster Response Planning

Chairman: Susan McHenry

Moderator: Sergio Magalini

Friday, 15 September, 09:00-10:30: Theatre 2

S 16.1

A Disaster Management Course for Senior Medical Officers and Administrators

Jacov Adler

Director, Emergency Department, Shaare Zedek Medical Centre, Jerusalem, Israel

During five weeks in the late summer of 1988, the First Disaster Management Course was held in Jerusalem. The course was attended by 23 senior medical officers and administrators from the following 23 countries: Central Africa-8; Central \& South America-9; Central Asia-6. The objectives of the course were twofold: 1) to present an overview of the causes of natural and man-made disasters, stressing man's effects on the ecology; and 2) to discuss the short and long term psychosocial and health aspects of disasters, and to introduce epidemiological parameters for their assessment and monitoring. The students visited the following national authorities and emergency services: The Civil Defence Training Centre; The National Emergency Economics Authority; The Magen David Ambulance Services and Blood Bank; The Fire Services Training Centre; and The National Geophysical Institute. The role and functions of all emergency services were presented and discussed, stressing the relations between the services and their integrated operation in disasters. Planning activities at all levels: national, regional, and local were presented, discussed, and exercised in workshops with each participant being assigned a specific task within his group. Several large-scale exercises in planning and disaster management were conducted, monitored by faculty members with practical experience in the following fields: emergency services; preventive medicine, and hospitalization to earthquake victims in a large city; planning of relief operations for a large rural population in Africa affected by famine and communicable diseases; and formulating a hospital emergency plan and implementing the plan in receiving casualties in an armed conflict. The participants were encouraged to present their views based on their experiences in their countries. The course proved to be an interesting learning experience for both the students and the faculty. Lessons learned from the course and from the participants' feedback in their respective countries will enable us to plan the organization of the second course for 1990 .
S 16.2

Planning National Medical Responses to a Mass Disaster Ermesto Pretto, Peter Safar

International Resuscitation Research Center, Department of Anesthesiology/Critical Care Medicine, Presbyterian-

University Hospital, University of Pittsburgh, Pittsburgh, Pennsylvania, USA

The primary goal of any National Disaster Medical Service (NDMS) must be to save lives in a mass disaster. Based on prior studies, in order to achieve this goal, NDMS response must be timely, appropriate, and efficient. We suggest a framework for NDMS patterned after the neural reflex arc, to include: 1) early warning (sensors); 2) flexible communication capability both horizontal and vertical (afferent limb);3) command and control (CNS integration); and 4) deployment (efferent limb). In order to fulfill these objectives, the organizational and resuscitation components which must be in place before the event include: recognition; public education; civilian-military collaborative responses; unified structure and command with pre-established modus operandi; immediate scouting-reporting of resuscitation needs; pre-designated trauma centers with medical hospital teams as well as equipped, self-sustaining field teams (mobile units), both on call 24 hours; and field resuscitation teams which can reach the scene within 6-12 hours. Disaster resuscitology components are: 1) life-supporting first aid (LSFA) by lay persons; 2) novel extrication; 3) ATLS by teams of paramedics and physicians; and 4) resuscitative surgery at the scene and in special hospitals. NDMS planning in the United States needs inclusion of resuscitation and wider discussion. 
Plenary Session 09

Mass Casualties, Civilian Aftermath

Chairman: Remi Russbach

Moderator: Eric Noji

Friday, 15 September, 11:00-12:30: Theatre 1

\section{P 9.2}

The International Medical Corps Experience Inside Afghanistan: Patterns of War Injuries, 1986-1987 John H Sloan

Harborview Medical Center, Seattle, Washington, USA

According to U.N. sources, 1.2-1.5 million people have died and many more have been injured during the 10 year Soviet-Afghan War. The International Medical Corps (IMC) created a medic training program for Afghans, with the intention of training a group of highly committed medics capable of diagnosing and treating $75 \%$ of medical diseases and basic surgical problems encountered. During the eight months of training supervised by Afghan, United States, and Canadian physicians and surgeons, the medics learn the basics of wound care and debridement, and how to perform extremity amputations, and chest injury drainage techniques. No intra-abdominal procedures are taught. Since there is no in-country referral system, they must be able to function independently. Once graduated, the medics staff clinics inside rural Afghanistan; typically clinics in protected areas such as caves. The medics are provided with major and minor surgical, simple orthopedic, and dental sets, and cloth gowns, masks, and gloves. An injury disease surveillance system was created for use at IMC clinics and medics have recorded data on 21,893 patient encounters from December, 1986 to August, 1987. Of the total patients, 1,488 or seven percent were injured. The vast majority of injuries were due to bombs or gunshot wounds, with a smaller number of injuries due to land mines or anti-personnel devices. About $70 \%$ of wounds involved an extremity, and wound debridement was the most common procedure performed. The case infection rate was $8.5 \%$. The case fatality rate of the study sample was $5.0 \%$. These findings compare favorably with data from prior conflicts. Currently, IMC medics staff over 40 rural clinics inside Afghanistan and are seeing in excess of 20,000 patients per month. Based on our analysis, we conclude that the trauma care of IMC medics resulted in relatively low morbidity and mortality among injured patients. This occurred in the choatic setting of war. The IMC training program may have broader uses in other conflicts and disasters. 


\section{P 9.3}

\section{Medical and Surgical Problems of the Nepal Earthquake \\ Niel Ineson, Maj, Peter Guy, Lt Col, Ronnie Baillie, Maj \\ Royal Army Medical Corps, British Military Hospital, Dharan, Nepal, BFPO 4}

On Sunday, 21 August 1988, a 45-second earth tremor hit east Nepal. The British Military Hospital (BMH) in Dharan treated 888 casualties from that disaster, 450 within the first 18 hours.

The vast majority of patients were treated as outpatients with only 260 requiring admission. Of the 628 treated as outpatients, most had bruising, lacerations, or simple fractures. Of the 260 admitted, the most common injuries were fractures of the lower leg (41), spinal injury (40), fractured femur (38), upper limb fractures (32), crushed chest (31), and pelvic fractures (31). In the five weeks immediately after the disaster, a total of 330 operative procedures were performed during 256 operations. All but fifty operations were under general anesthetic. An intensive care unit was established to cope with the seriously injured and for several cases of tetanus which occurred in injured patients who presented late and had not received adequate primary wound cleaning. As in many major disasters, the threat of infectious disease after the earthquake was considerable. The camp and town water supply was monitored for chlorine content and bacteria by the hospital laboratory. No serious pathogens were isolated and no significant increase in the background level of diarrheal illness occurred. In Dharan town, at least 130 people were killed. Of the 888 treated at the BMH, only 10 died.

\section{P 9.4}

Ten Years of War along Thai-Cambodian Border: A Medical Review Paul R Shakti

UNBRO, United Nations Building, Bangkok, Thailand

Decades of war in Cambodia left millions dead and homeless; many thousands fled the country. Since 1982, United Nations Border Relief Operation (UNBRO) has been providing humanitarian assistance to approximately 300,000 displaced Cambodians encamped along the Thai-Cambodian border. Due to severity of war, many thousands died from war injuries; thousands handicapped or disabled people are living in the camps. In some groups, up to $10 \%$ of all population are the disabled. But, war injuries are not the only cause of death or disablement in this population. Endemic entities, malaria, measles, poliomyelitis, diarrhea, and nutritional problems killed many more during the early stage of the refugee influx. However, UNBRO, with the generous assistance of the international donors' community, has been able to manage and contain these problems to a very satisfactory level. The mental health of the population is deteriorating gradually. Years of idleness, confinement, hopelessness, and persistent insecurity have eroded the traditional culture and socio-familial structures. The only reasonable solution to this Cambodian problem is the safe return of these people to a peaceful Cambodia 
Plenary Session 10

Armenia: The Lessons

Chairmen: Peter Safar, Victor Semenov

Moderator: Andrei Kisselev

Friday, 15 September, 13:45-15:45: Convention Hall

\section{P 9.5}

Late Management of War-Injured Patients with Special

Reference to Reconstructive Surgery

Richard R Hammer

Professor, Department of Orthopedic Surgery, University

Hospital, Linkoping, Sweden

During the period December 1986 to May 1988, a total of 130 patients with sequalae consequent upon either war injuries or torture were referred to our hospital. Patient catchment was predominantly from the Middle East with lesser numbers from several countries in Latin America. A striking feature was the significantly enhanced limb salvage in patients from the Middle East. This correlated with the use of various types of external fixation systems. By contrast, in Latin America, the lack of such systems has led to a high incidence of early amputations, particularly in patients with high velocity injuries caused by missiles or mine explosions. In this group of patients, only 10 could be fitted directly with a below-knee prosthesis; the remaining 12 had a total of 15 surgical procedures, the most common being removal of one or more amputation neuromas. Eight patients from the Middle East had massive bone defects, chronic osteomyelitis and compromised soft tissue caused by high velocity missiles or mine explosions. Prior to referral, the duration of osteomyelitis ranged from 9 to 36 months and the number of unsuccessful surgical attempts to cure infection and effect wound closure ranged from 2 to 9 (mean=4). The most common procedure had been sequestrectomy, usually insufficient, followed by repeated grafts of cancellous bone and split skin grafts. All these patients were treated successfully in our clinic with extended sequestrectomy, implantation of Gentamycin beads, and local pedicle or free vascular flap. In six patients, this was followed, 4-8 weeks later, by massive cancellous bone graft or free vasculous bone graft. All patients healed and no recurrence of infections has been registered. It is concluded that primary stabilization with an external fixation system increases the probability of limb salvage in combat injuries. Furthermore, in late management of long-standing, post-traumatic osteomyelitis, aggressive and extended sequestrectomy combined with modern microvascular techniques are keys to successful outcome.

\section{P 10.5}

Resuscitation Potentials in Major Earthquakes. Methodology of USA-USSR Site Visit Study after Armenia Disaster of December 7, 1988

Peter Safar, Victor Semenov, E Ricci, Miroslav Klain, David Crippen, Ernesto Pretto, S Tisherman, L Comfort, JAbrams, et al. International Resuscitation Research Center (IRRC), Schools of Public Health and Engineering, University of Pittsburgh; and Institute of Reanimatology, USSR Academy of Medical Sciences, Moscow

Preparations for mass disasters lack resuscitation response (basicadvanced-prolonged life support [BLS-ALS-PIS], resuscitative surgery). Our previous interviews of earthquake survivors in Italy, Peru, and Mexico suggested that some people killed died slowly and were accessible and resuscitatable. Hypotheses: BLS (life-supporting first aid, LSFA) within minutes, triage, ALS by medical teams within less than six hours, and PLS during transport and resuscitative surgery can significantly reduce mortality.

We developed plans and instruments which utilized EMS evaluation methods (protocol, procedure manual, interview questionnaires, check lists for record research) for use by a multiprofessional team to conduct a site visit study in Armenia in March 1989. The questions asked of eyewitnesses (victims, families, rescuers, nurses, physicians, administrators) included: number injured; resources applied; accessibility; extrication; dying patterns; causes of death; BLS and ALS available and used; and potentials and time factors. Records to be researched include autopsy. Summary, conclusions and recommendations will be presented to WAEDM Congress delegates for input. 
Special Presentation 01

Time and location to be announced

\section{P 10.6 \\ Mortality and Morbidity Following the 1988 Earthquake in Soviet Armenia Enic $K$ Noji \\ Johns Hopkins Hospital, Baltimore, Maryland, USA}

An earthquake registering 6.9 on the Richter scale hit the northern part of the Armenian Republic of the Soviet Union on 7 December 1988 resulting in thousands of deaths and injuries. This study was undertaken: (1) to determine earthquake-related injury patterns and severity; (2) assess factors associated with survival vs. death; and (3) to identify the most appropriate type and timing of rescue. The study drew upon a population-based survey of three towns seriously affected by the earthquake. Data collected on site by the author during the period immediately after the earthquake was obtained from field surveys of the devastated towns, direct interviews with survivors and officials of the Armenian Ministry of Health, and from data collected by the Division of Information Systems, Armenian Ministry of Health. Deaths were 68 times and injury rates seven times higher in trapped than in non-trapped victims. The possibility of escape was crucial for survival and depended on the type of building and occupant escape behavior. During the first 24 hours, $89 \%$ of persons found alive were rescued. Most of the initial rescue work and medical relief was totally disorganized and entirely carried out by unprepared local people. The first outside assistance from Soviet authorities did not arrive for 24 hours. No international assistance was available for several days. Crush syndrome due to limb compression became a major problem with 23 patients developing acute renal failure requiring dialysis. Since all hospitals in the region were severely damaged, these patients were transferred to hospitals in unaffected parts of the country or to neighboring Soviet Georgia. Analysis of the data suggests that with few exceptions, the emergency phase for medical care was limited to the three to four days after impact. These results point to the importance of wellorganized, local, disaster preparedness capabilities since outside assistance invariably will arrive too late for effective life-saving.
SP 1.1

Prehospital Transcutaneous Pacing by Paramedics and Emergency Medical Technicians (EMTs): Clinical and System Effectiveness

Judith Reid Graves and Richard O Cummins

Department of Medicine, University of Washington; Center for

Evaluation of Emergency Medical Services; Emergency Medical Services Division; Seattle-King County Department of Public

Health; Seattle, Washington, USA

\section{PURPOSE:}

This project has four aims: (1) implement transcutaneous pacing throughout an urban-suburban EMS system; (2) determine the need for pacing for bradycardic patients; (3) establish the effectiveness of pacing for asystolic cardiac arrest patients performed by paramedics; and (4) by EMTs.

\section{METHODS:}

71 paramedics in 8 paramedic units, and 375 EMTs in 21 fire districts were trained to use stand-alone, transcutaneous pacemakers. Protocols instructed the paramedics to initiate transcutaneous pacing for patients with significant bradycardia unresponsive to atropine, and for patients in cardiac arrest with asystole. EMTs began pacing for patients in either primary or post-shock asystole.

\section{RESULTS:}

In one year, 358 cardiac arrest patients have been paced in the prehospital setting ( 285 by paramedics, and 63 by EMTs); paramedics also paced 28 patien ts with bradycardia, not in cardiac arrest. 288 of the $358(80 \%)$ expired at the scene; $31(9 \%)$ expired in casualty; and $34(9 \%)$ died in the hospital. The number discharged alive was $5(2 \%)$. Average duration of pacing was $15 \mathrm{~min}$; average time from collapse to pacing by paramedics was 18 minutes; and by EMTs, 8 minutes. Of the 28 patients paced for bradycardia, 24 patients responded and were paced to casualty. 19 of these $24(80 \%)$ were admitted to the hospital; $15(63 \%)$ received temporary transvenous pacemakers; $14(50 \%)$ were discharged alive, 9 with temporary transvenous pacers placed, and 2 with permanent pacemakers. Similar urban-suburban systems can expect to encounter 5 bradycardic patients who need prehospital pacing per 100 cardiac arrests per year.

\section{CONCLUSIONS:}

We conclude that transcutaneous pacing can be implemented effectively in EMS systems. So far, late pacing by paramedics for cardiac arrest patients has not been associated with improved survival. However, earlier pacing by EMTs displays promise. Early pacing was a life-sustaining intervention for some patients with bradycardia as shown by the initial clinical response, and the need for later transvenous pacing. Transcutaneous pacing for bradycardias alone, may justify the addition of this therapy to paramedicbased systems. Further study is necessary to determine if transcutaneous pacing by EMTs merits widespread implementation. 